\title{
Unusual mechanism of vortex viscosity generated by mixed normal modes in superconductors with broken time reversal symmetry
}

\author{
Mihail Silaev ${ }^{1,2,3,4}$ and Egor Babaev ${ }^{3,4}$ \\ ${ }^{1}$ O. V. Lounasmaa Laboratory, P.O. Box 15100, FI-00076 Aalto University, Finland \\ ${ }^{2}$ Institute for Physics of Microstructures RAS, 603950 Nizhny Novgorod, Russia. \\ ${ }^{3}$ Department of Theoretical Physics, The Royal Institute of Technology, Stockholm, SE-10691 Sweden \\ 4 Department of Physics, University of Massachusetts Amherst, MA 01003 USA
}

(Dated: September 26, 2018)

\begin{abstract}
We show that under certain conditions multiband superconductors with broken time-reversal symmetry have a new vortex viscosity-generating mechanism which is different from that in conventional superconductors. It appears due to the existence of mixed superfluid phase-density mode inside vortex core. This new contribution is dominant near the time reversal symmetry breaking phase transition. The results could be relevant for three band superconductor $B a_{1-x} K_{x} F e_{2} A s_{2}$.

PACS numbers: 74.25.QP, 74.25.Fy, 73.40.Gk
\end{abstract}

Recent discoveries of many novel multiband superconducting compounds have motivated the current quest of theoretical understanding of their basic properties. Especially strong impact has the recent discovery of iron based superconductors [1]. Namely it was discussed in [2, 3] that such superconductors can break time reversal symmetry because these system can have frustrated ground state values of the order parameter phase differences in different bands $\theta_{i k}=\theta_{i}-\theta_{k} \neq \pi n$. In that case a ground state has a broken time reversal symmetry (BTRS) which is associated with the complex conjugate of the order parameter $\psi \rightarrow \psi^{*}$. Therefore such superconductors break $U(1) \times Z_{2}$ symmetry [4]. Physically this implies existence of persistent "Josephson current" between the three bands which is different for two ground states. It was recently demonstrated that such physics very likely occurs in strongly hole doped $B a_{1-x} K_{x} F e_{2} A s_{2}$ [5]. Alternatively the other scenarios of time reversal symmetry breakdown in iron-based superconductors have been discussed recently [6].

In this kind of BTRS state there appear new phenomena which are absent in conventional and even extended $s$ wave multiband superconductors. Indeed it has been shown to support a new kind of topological defects - $C P^{2}$ skyrmions [7], Leggett's mode which becomes massless at the $Z_{2}$ phase transition [8], as well as mixed phase-density collective modes in $U(1) \times Z_{2}$ state [4]. Moreover even in the $U(1)$ frustrated systems, time reversal symmetry breakdown can occur inside vortex excitations [4]. Beyond the mean field approximation and for sufficiently strong frustration of interband interactions such systems can have an unusual normal state which breaks $Z_{2}$ symmetry as a precursor to a superconducting phase transition [9].

Since the system can break time reversal symmetry at certain doping [5], the superconducting state in the immediate vicinity of the time reversal symmetry breaking phase transition should be very interesting because of the existence of a diverging length scale associated with the
$Z_{2}$ symmetry breakdown. In this paper we show that the BTRS superconducting state with vortices has highly unusual thermodynamic and transport properties near the $Z_{2}$ symmetry breaking transition. The peculiarities of vortex state can be helpful to obtain experimental identification of BTRS superconductivity in particular compounds.

We employ the three band Ginzburg-Landau (GL) expansion of the free energy density

$$
\begin{array}{r}
f=\sum_{k=1}^{3}\left[\alpha_{k}\left|\psi_{k}\right|^{2}+\frac{\beta_{k}}{2}\left|\psi_{k}\right|^{4}+g_{k}\left|(\nabla+i \mathbf{A}) \psi_{k}\right|^{2}\right]+ \\
\sum_{i, k=1}^{3} \gamma_{i k} \psi_{i} \psi_{k}^{*}+\text { c.c. }+|\nabla \times \mathbf{A}|^{2} / 8 \pi
\end{array}
$$

Here, $\psi_{k}$ are the order parameters in each band labelled by band index $k=1,2,3$ and the second term is interband Josephson coupling energy characterized by interband coupling constants $\gamma_{i k}$. The field $\mathbf{A}$ is vector potential. For formal microscopic justification of multiband GL functionals see [10], GL expansion for three band BTRS superconductor was recently studied in detail in [5] where it was shown that the doping level $x$ in $B a_{1-x} K_{x} F e_{2} A s_{2}$ determines the interband pairing interaction between electron and hole pockets $u_{h e}$. The relation to our parameters is following $\gamma_{13}=u_{h e}$ and $\gamma_{12}=\gamma_{23}=u_{h h}$ which is the interaction between hole pockets. Such an expansion may contain also other terms which however will not change quantitatively conclusions of this paper, thus we choose to work with the minimal model.

First we investigate the equilibrium vortex structures in three band superconductor. We substitute the order parameters to GL equation in the form $\psi_{k}=\Delta_{k} e^{i \theta_{k}}$ where $\Delta_{k}$ is real and separate the real and imaginary parts introducing the gauge invariant superfluid velocities $\mathbf{Q}_{\mathbf{k}}=\mathbf{A}+\nabla \theta_{k}$. It should be noted that even when a ground state has only $U(1)$ broken symmetry, the GL 
model (11) allows for topological excitations with phase differences of order parameter components $\theta_{i k}=\theta_{i}-\theta_{k} \neq$ $\pi n$ [4]. In the particular case of axially symmetric single vortex in BTRS phase this results in the radial dependence of the order parameter phases $\theta_{i k}=\theta_{i k}(r)$ (we assume that vortex center is at the origin $r=0$ ).

Thus the additional degree of freedom due to the frustrated phase difference in three component system allows for a static mixed phase-density mode which appears inside vortex cores in BTRS phase. To explore its impact on the vortex physics we employ the minimal model which in particular describes possible BTRS transition to the $s+i s$ state in $B a_{1-x} K_{x} F e_{2} A s_{2}$ [5].

Consider a single vortex in three component superconductor described by GL functional (1) with $\alpha_{1}=\alpha_{3}=\alpha$ and $\beta_{1}=\beta_{3}=\beta, g_{1}=g_{3}=g$. To study the modification of vortex properties during he BTRS transition we fix the values of $\gamma_{12}=\gamma_{23}=\gamma$ and vary $\gamma_{13}$ which models the electron-hole interaction determined by the level of doping $x$ in $B a_{1-x} K_{x} F e_{2} A s_{2}$ compound [5]. Qualitatively our conclusions will however be valid also for a non-symmetric set of couplings.

For such choice of GL coefficients we will use an ansatz for vortex solutions $\theta_{12}=\theta_{23}=\theta(r)$ and $\Delta_{1}=\Delta_{3}=$ $\Delta(r)$. The GL equations in this case read

$$
\begin{gathered}
g\left[\nabla_{r}^{2} \theta+2(\ln \Delta)_{r}^{\prime} \theta_{r}^{\prime}\right]+\frac{\gamma \Delta_{2}}{\Delta} \sin \theta+\gamma_{13} \sin (2 \theta)=0 \\
{\left[g_{2}\left(\nabla_{r}^{2}-\mathbf{Q}_{\mathbf{2}}^{2}\right)-\alpha_{2}-\beta_{2} \Delta_{2}^{2}\right] \Delta_{2}=2 \gamma \Delta \cos \theta} \\
{\left[g\left(\nabla_{r}^{2}-\mathbf{Q}^{2}\right)-\alpha-\gamma_{13} \cos (2 \theta)-\beta \Delta^{2}\right] \Delta=\gamma \Delta_{2} \cos \theta}
\end{gathered}
$$

where $\mathbf{Q}^{2}=(A+1 / r)^{2}+\theta_{r}^{\prime 2}$ and $\mathbf{Q}_{\mathbf{2}}^{2}=(A+1 / r)^{2}$. For the vector potential we use a radial gauge $\mathbf{A}=$ $A(r)(-\sin \alpha, \cos \alpha)$.

First let us consider the modification of asymptotical properties of the system (2) far from the vortex center during the BTRS transition. At small couplings $\gamma_{13}<\gamma_{13}^{*}$ the system is in the plain $U(1)$ symmetry breaking state with the relative phase between superconducting components $\theta_{12}=\pi$. The critical value of coupling separating the $U(1)$ and $U(1) \times Z_{2}$ bulk phases is given by $\gamma_{13}^{*}=\gamma \Delta_{02} / 2 \Delta_{0}$, where $\Delta_{0}$ and $\Delta_{20}$ are bulk values of the amplitudes $\Delta$ and $\Delta_{2}$. Beyond the threshold $\gamma_{13}>\gamma_{13}^{*}$ the time reversal symmetry is broken so that $0<\theta_{12}<\pi$. This behavior of bulk $\theta_{12}$ is shown in Fig. (1) a by blue dashed curve.

The masses of symmetric mixed modes obtained by the linearization of the system (2) are shown in Fig.(1)a as functions of the coupling $\mu=\mu_{0,1,2}\left(\gamma_{13}\right)$. In general the three band GL model (10) has five distinct mixed modes which are the fundamental solutions of linearized equations [4]. The masses $\mu_{3,4}$ correspond to non-symmetric modes with $\Delta_{1}(r) \neq \Delta_{3}(r)$ and $\theta_{12}(r) \neq \theta_{13}(r)$.

In $U(1)$ phase when $\gamma_{13}<\gamma_{13}^{*}$ the mode shown by red solid line is a pure phase one which is decoupled from order parameter densities. However in general it can still be excited inside vortex core due to nonlinearities. In this case $Z_{2}$ symmetry can be broken locally in the core but not in the bulk [4]. At the critical point of $Z_{2}$ symmetry breakdown in the bulk of the system the mixed mode has zero mass $\mu_{0}\left(\gamma_{13}^{*}\right)=0$ [11].

At $Z_{2}$ critical point, existence of massless mode results in a power-law localization of vortex-core solutions. Note that in this case the anharmonism in Eq.(2) is important. Here at large $r$ the field deviations from bulk values can be found in the form of power law expansions (the details of asymptotic analysis are given in Supplementary material) $\left(\tilde{\Delta}, \tilde{\Delta}_{2}, \tilde{\theta}\right)=\left(C_{\Delta} / r^{2}, C_{\Delta 2} / r^{2}, C_{\theta} / r\right)$.

Now let us search numerically for vortex solutions of Eqs. (21) to show that the vortex energy and viscosity have anomalies at $Z_{2}$ phase transition. As will be discussed below, it is important to take into account the following three circumstances for the accurate description of this anomalous behavior at the BTRS phase transition (i) phase-density modes mixing, (ii) appearance of massless mode and (iii)anharmonism in Eqs.(2).

To find possible vortex structures we implement a numerical solution of the full GL system (2) supplemented by the Ampere's law for magnetic field. To define the boundary conditions for the fields we consider a vortex lattice and thus use the circular cell approximation (see also remark [12]). At the boundary of the Weigner-Seits cell $r=r_{s}$ the fields satisfy $\Delta^{\prime}=\Delta_{2}^{\prime}=\theta^{\prime}=0$ and $r A+1=0$. The former determines the order parameter to be periodic function and the latter one provides magnetic flux quantization. Also from the first of Eqs.(2), it follows that the boundary condition for the phase is $\theta_{r}^{\prime}(r=0)=0$.

We investigated the vortex structure as function of interband coupling $\gamma_{13}$ which is determined by the doping level in $B a_{1-x} K_{x} F e_{2} A s_{2}$ compound [5]. We have found that the relative phases of the order parameter components in BTRS superconductor $\gamma_{13}>\gamma_{13}^{*}$ always have non-trivial variation $\theta_{12}=\theta_{23}=\theta(r)$ in contrast to the usual time-reversal invariant case. Examples of phase distributions are shown in Fig.(1)c for a set of $\gamma_{13}$ values decreasing towards the vortex core transition point $\gamma_{13}^{c}$ which will be discussed below. We will see that such phase variation produces an additional friction force on the moving vortices.

For $\gamma_{13}>\gamma_{13}^{*}$ the vortex solution is unique. Its energy is shown in Fig.(11)b by solid red curve denoted as Branch 2. Even at the point of bulk $Z_{2}$ transition the energy remains finite due to the discussed above anharmonism of the massless mixed mode in Eqs.(2). However its contribution provides a peak of vortex energy close to $\gamma_{13}=\gamma_{13}^{*}$ (where this mode becomes massless).

On the other hand time reversal invariant state at $\gamma_{13}<\gamma_{13}^{*}$ can support two different vortex structures. To demonstrate it we note at first that the Eqs.(2) always have solution with the relative phase $\theta=\pi$. We find that this solution is stable in $U(1)$ domain. At the same range of parameters the $Z_{2}$ symmetry can be broken 
in the vortex core leading to the non-trivial variation of $\theta(r)$ with asymptotic boundary condition $\theta(r \rightarrow \infty)=\pi$. We find numerically that these vortex structures can coexist at a certain region $\gamma_{13}<\gamma_{13}^{*}$ (note that one of the solutions can be unstable [13] in the coexistence region, but this does not affect conclusions of this paper). The corresponding branches of vortex energy are shown in Fig. (10). There is a critical value of interband coupling $\gamma_{13}^{c}<\gamma_{13}^{*}$ where the two branches merge. This critical coupling is determined as the eigenvalue of linearized first equation in the system (2) which we write in the form of Sturm-Liouville equation $\hat{L} \tilde{\theta}=2 \gamma_{13}^{c} \Delta^{2} \tilde{\theta}$ where $\tilde{\theta}=\pi-\theta$ and $\hat{L}=-g\left[\Delta^{2} \nabla_{r}^{2}+\left(\Delta^{2}\right)_{r}^{\prime} \partial_{r}\right]+\gamma \Delta_{2} \Delta$ is a hermitian operator. This means (see Supplementary Material) that at $\gamma_{13}>\gamma_{13}^{c}$ the amplitude of relative phase variation is given by $\tilde{\theta} \sim \sqrt{\gamma_{13}-\gamma_{13}^{c}}$ so that the energy difference between Branch 1 and Branch 2 is linear $\varepsilon_{2}-\varepsilon_{1} \sim \gamma_{13}-\gamma_{13}^{c}$.

The obtained BTRS modification of vortex core structure is manifested transport properties determined by vortex viscosity. To describe a non-equilibrium process of vortex motion, we use time-dependent GL model (TDGL) (for a review of TDGL approach see e.g. [14, 15]) generalized to a multiband case

$$
\Gamma_{k}\left(\partial_{t}-i \varphi\right) \psi_{k}=-\frac{\delta}{\delta \psi_{k}^{*}} \int f d^{3} \mathbf{r}
$$

where $k=1,2,3, \Gamma_{k}$ are damping constants and $\varphi$ is the potential of a quasistationary electric field. Choosing Couloumb gauge for the vector potential $\operatorname{div} \mathbf{A}=0$ we obtain the Poisson equation (see Supplementary material for detailed discussion)

$$
\sigma_{n} \triangle \varphi=2 \sum_{k=1}^{3} \Gamma_{k} \Delta_{k}^{2}\left(\varphi-\dot{\theta}_{k}\right)
$$

where $\sigma_{n}$ is a normal state electric conductivity. Equation (4) will be employed to calculate the distribution of electric field generated by a moving vortex.

Vortex motion introduces a distortion of the order parameter and vector potential fields. For a slow vortex motion with a given velocity $\mathbf{U}$ we calculate the time dependence by making Galilean transformation $\mathbf{r} \rightarrow \mathbf{r}-\mathbf{U} t$ of equilibrium fields so that $\partial_{t}=-(\mathbf{U} \cdot \nabla)$. We now assume that $\mathbf{U}=U \mathbf{x}$ and search for the electrostatic potential in the form $\varphi=U\left[\varphi_{\alpha}(r) \sin \alpha-\varphi_{r}(r) \cos \alpha\right]$. The resulting equations read

$$
\begin{gathered}
\sigma_{n}\left(\nabla_{r}^{2}-1 / r^{2}\right) \varphi_{\alpha}=2 \sum_{k=1}^{3} \Gamma_{k} \Delta_{k}^{2}\left(\varphi_{\alpha}-1 / r\right) \\
\sigma_{n}\left(\nabla_{r}^{2}-1 / r^{2}\right) \varphi_{r}=2 \sum_{k=1}^{3} \Gamma_{k} \Delta_{k}^{2}\left(\varphi_{r}+\theta_{k}^{\prime}\right)
\end{gathered}
$$

where $\theta_{k}^{\prime}=\partial \theta_{k} / \partial r$. Note that in Eq.(6) the derivatives $\theta_{k}^{\prime}$ can be expressed through the two functions $\theta_{12}(r)$ and $\theta_{13}(r)$ using the condition for the radial current to be zero

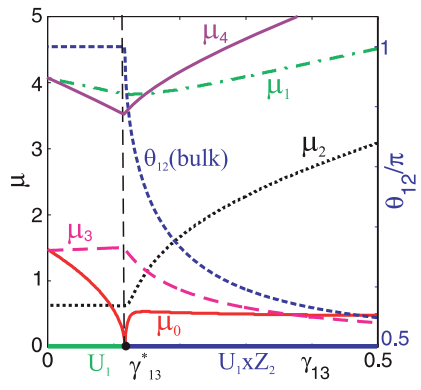

(c) Phase distribution

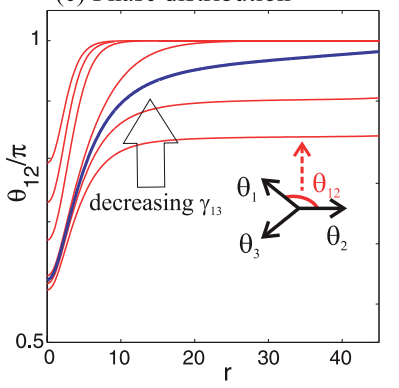

(a) Masses of linear modes (b) Vortex energy
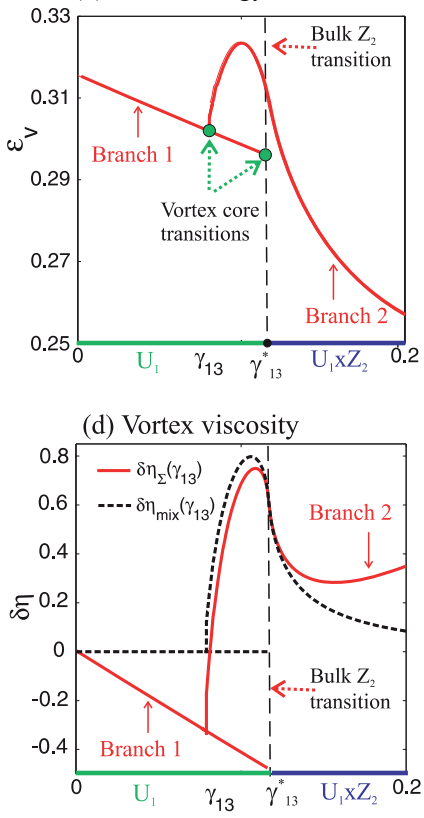

FIG. 1: (a) Masses of the asymptotic mixed modes of the system (2). The GL parameters are $\alpha_{1}=\alpha_{2}=\alpha_{3}=-0.5$, $\beta_{1}=\beta_{2}=\beta_{3}=10, \gamma_{12}=\gamma_{23}=0.2, g_{2}=5$ and $g=0.1$. The modes $\mu_{0,1,2}$ corresponds to symmetric excitations with $\Delta_{1}=\Delta_{3}$. The modes $\mu_{3,4}$ break this symmetry. By dashed blue line in (a) the ground state (bulk) phase difference is shown. (b) Two branches of vortex energy. Branch 1 corresponds to the vortex solutions which do not break time reversal symmetry. Branch 2 corresponds to the solutions with non-homogeneous relative phase (i.e. BTRS solutions). (c) Relative phase distribution $\theta_{12}=\theta_{23}=\theta(r)$ inside vortex core corresponding to the Branch 2. For decreasing $\gamma_{13}$ one first meets the second order phase transition in bulk where the characteristic scale of variation of $\theta_{12}(r)$ is the largest (blue curve). At the critical value of $\gamma_{13}=\gamma_{13}^{c}$ the Branches 1 and 2 merge when the amplitude of $\theta_{12}=\theta_{12}(r)$ decreases to zero as $\sqrt{\gamma_{13}-\gamma_{13}^{c}}$. (d) Vortex viscosity variation $\delta \eta\left(\gamma_{13}\right)=\eta\left(\gamma_{13}\right)-\eta(0)$ (red solid line is total viscosity and black dashed line is mixed mode contribution).

$\sum_{k} g_{k} \Delta_{k}^{2} \theta_{k}^{\prime}=0$. In the circular cell approximation the boundary condition require the tangential component of the electric field to be zero $\left.\left(\mathbf{E} \cdot \mathbf{e}_{\alpha}\right)\right|_{r=r_{s}}=0$. Recalling that $\dot{\mathbf{A}}=-U \cos \alpha A^{\prime} \mathbf{e}_{\alpha}$ we obtain at $\varphi_{\alpha}\left(r_{s}\right)-r_{s} A^{\prime}\left(r_{s}\right)=$ 0 and $\varphi_{r}\left(r_{s}\right)=0$. Also from the Eqs.(516) follows that $\varphi_{\alpha}(r=0)=\varphi_{r}(r=0)=0$.

At first we note that Eq. (15) coincides with that for the vortices in time reversal invariant superconductors (see e.g. [14, 15]). It determines Bardeen-Stephen vortex viscosity [16]. The second Eq.(6) determines qualitatively new part of the scalar potential which appears due to the phase-density mixed mode in BTRS superconductor. The source in the r.h.s. of this equation is determined by the radial dependencies of the relative phase $\theta_{12}(r)$ [example is shown in the Fig.(11)c].

Consider now electric field distribution generated by 
a moving vortex. The electric field can be written as a superposition of two terms $\mathbf{E}=\mathbf{E}_{\alpha}+\mathbf{E}_{\mathbf{r}}$ where $\mathbf{E}_{\alpha}=$ $U\left[\nabla\left(\varphi_{\alpha} \sin \alpha\right)-\cos \alpha A_{r}^{\prime} \mathbf{e}_{\alpha}\right]$ and $\mathbf{E}_{\mathbf{r}}=U \nabla\left(\varphi_{r} \cos \alpha\right)$. The first term $\mathbf{E}_{\alpha}$ here is a usual dipole-like field induced around moving vortex. The second term $\mathbf{E}_{\mathbf{r}}$ is the mixed mode contribution which exists only in BTRS superconductors.

Distributions of $\mathbf{E}_{\alpha}$ and $\mathbf{E}_{\mathbf{r}}$ components of the electric field are shown in the Fig.(2) a,b. From Fig.(2) a one can see that the component $\mathbf{E}_{\alpha}$ determines the average electric field in the sample $\langle\mathbf{E}\rangle=\left(\pi r_{s}^{2}\right)^{-1} \int_{u . c .} \mathbf{E}_{\alpha} d^{2} r=$ $[\mathbf{B} \times \mathbf{U}]$ where $\mathbf{B}$ is the average magnetic induction. The other component $\mathbf{E}_{\mathbf{r}}$ shown in Fig.(2) b does not contribute to the average $\left\langle\mathbf{E}_{\mathbf{r}}\right\rangle_{\text {u.c. }}=0$.

The relation between vortex velocity $\mathbf{U}$ and transport current $\mathbf{j}_{\mathbf{t r}}$ is in general determined by the balance of the forces acting on the moving vortex. There are two of them: Lorentz force from the transport current and the force from the environment $\mathbf{f}_{\mathbf{e n v}}$ given by the expression (under the assumption that London penetration length is much larger than the vortex core size)

$$
\mathbf{f}_{\mathbf{e n v}}=2 \sum_{k=1}^{3} \int \Gamma_{k}\left(\nabla \Delta_{k} \dot{\Delta}_{k}+\Phi_{k} \mathbf{Q}_{\mathbf{k}} \Delta_{k}^{2}\right) d^{2} \mathbf{r}
$$

where we introduced gauge invariant scalar potential $\Phi_{k}=\varphi_{k}-\dot{\theta}_{k}$. To find a linear response of the environment we need to keep the terms in Eq.(7) up to the first order in vortex velocity. In this approximation the force from the environment provides viscous drag which has the general form $\mathbf{f}_{\mathbf{e n v}}=-\eta \mathbf{U}$ where $\eta$ is vortex viscosity. We find that in BTRS superconductor it can be presented as a superposition of three terms of different physical origin $\eta=\eta_{T}+\eta_{B S}+\eta_{\text {mix }}$. Here the first two terms appear in ordinary viscous vortex motion: these are the Tinkham [17] and Bardeen-Stephen [16] contributions. The third term $\eta_{m i x}$ is completely new and appears due to the electric mixed phase-density mode in the BTRS vortex core:

$$
\eta_{\text {mix }}=2 \pi \sum_{k=1}^{3} \Gamma_{k} \int_{0}^{\infty} \Delta_{k}^{2}\left[r \theta_{k}^{\prime}\left(\theta_{k}^{\prime}+\varphi_{r}\right)\right] d r
$$

where we put $r_{s}=\infty$ for well-separated vortices. The physical origin of viscosity (8) is the electric field excitation due to the mixed mode. The corresponding electric field pattern and charge density around moving vortex is shown in Figs.(2) b, d.

We calculated the total vortex viscosity $\eta$ by solving Eqs. (56) using the vortex structure determined by static GL Eqs.(2). We find a striking behavior of viscosity near the BTRS transition shown in Fig 1 d as function of interband coupling $\gamma_{13}$. The mixed mode contribution (shown by black dashed line) has a pronounced maximum near BTRS phase transition where the mixed mode becomes massless. The viscosity however still remains finite even
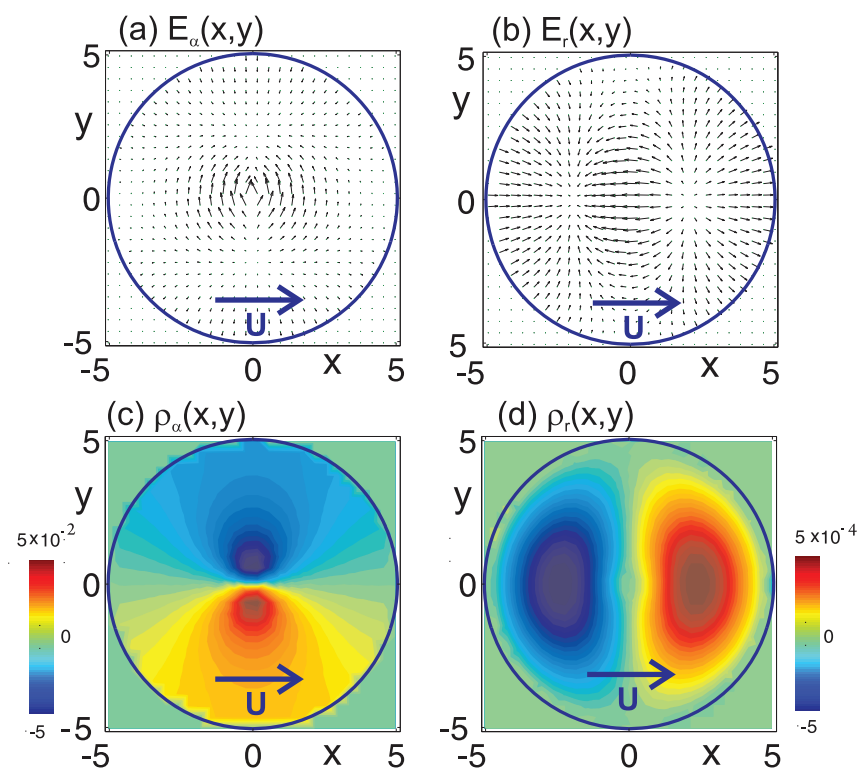

FIG. 2: (a,b) The distributions of (a) dipole-like $\mathbf{E}_{\alpha}$ and (b) induced by mixed mode $\mathbf{E}_{\mathbf{r}}$ parts of the total electric field (shown by arrows) $\mathbf{E}=\mathbf{E}_{\alpha}+\mathbf{E}_{\mathbf{r}}$ in the unit cell around the vortex moving with velocity $\mathbf{U}$. (c,d) The distribution of electric charge around the moving vortex. (c) $\rho_{\alpha}(x, y)=$ $\operatorname{div} \mathbf{E}_{\alpha} / 4 \pi$ which coincides with the charge in time reversal invariant superconductor. (d) $\rho_{r}(x, y)=\operatorname{div} \mathbf{E}_{\mathbf{r}} / 4 \pi$ appears in BTRS superconductor.By blue solid circle the boundary of circular cell is shown.

at the critical point due to the anharmonism in Eqs.(2) which provides a power-law decay for the phase-density mode contribution to viscosity as well as to the vortex energy . The conventional Tinkham and Bardeen- Stephen contributions are monotonic functions of $\gamma_{13}$. Summing up all contributions we find that here the behavior of the total viscosity is dominated by the mixed mode near the BTRS transition. It is shown by solid red line which features a pronounced peak. This anomalous behavior is realized for vortex structures belonging to Branch 2 with BTRS either in the bulk at $\gamma_{13}>\gamma_{13}^{*}$ or in the vortex core at $\gamma_{13}<\gamma_{13}^{*}$. On the other hand vortex solution without BTRS corresponding to the Branch 1 has monotonic viscosity. Comparing Figs 10 and 1 one can see that the vortex energy and viscosity behave rather similar as functions of $\gamma_{13}$.

In conclusion we reported a new mechanism contributing to vortex viscosity in BTRS superconductors. The results are generic for BTRS superconductors with mode mixing. In particular it is not specific to three-band superconductor but should also apply to BTRS states with different number of bands or different interband frustration which exhibit mode mixing (some of which were discussed in [18]). It leads to a pronounced anomaly at the phase transition where time reversal symmetry is broken. Thus one can potentially observe this phase transition by measuring the anomalous behavior of both thermody- 
namic properties (vortex energy $\varepsilon_{v}$ determines the lower critical field $\left.H_{c 1}=\varepsilon_{v} / \Phi_{0}\right)$ and transport properties such as flux flow resistance which is determined by vortex viscosity. It can be utilized to detect possible $s+i s$ state in $B a_{1-x} K_{x} \mathrm{Fe}_{2} A s_{2}$.

We thank Daniel Weston for discussions. MS was supported by the Swedish Research Council, Russian Foundation for Basic Research Grants No 11-02-00891, 13-02-97126 and Russian President scholarship (SP6811.2013.5), EB was supported by the US National Science Foundation CAREER Award No. DMR-0955902, and by Knut and Alice Wallenberg Foundation through the Royal Swedish Academy of Sciences, Swedish Research Council.

\section{SUPPLEMENTARY MATERIAL}

\section{Power law asymptotic of order parameter fields in zero mass regime}

We consider the system

$$
\begin{gathered}
g\left[\nabla_{r}^{2} \theta+2(\ln \Delta)_{r}^{\prime} \theta_{r}^{\prime}\right]+\frac{\gamma \Delta_{2}}{\Delta} \sin \theta+\gamma_{13} \sin (2 \theta)=0 \\
{\left[g_{2}\left(\nabla_{r}^{2}-\mathbf{Q}_{\mathbf{2}}^{2}\right)-\alpha_{2}-\beta_{2} \Delta_{2}^{2}\right] \Delta_{2}=2 \gamma \Delta \cos \theta} \\
{\left[g\left(\nabla_{r}^{2}-\mathbf{Q}^{2}\right)-\alpha-\gamma_{13} \cos (2 \theta)-\beta \Delta^{2}\right] \Delta=\gamma \Delta_{2} \cos \theta}
\end{gathered}
$$

where $\mathbf{Q}^{2}=(A+1 / r)^{2}+\theta_{r}^{\prime 2}$ and $\mathbf{Q}_{2}^{2}=(A+1 / r)^{2}$. For the vector potential we use the radial gauge $\mathbf{A}=$ $A(r)(-\sin \alpha, \cos \alpha)$.

We are interested in particular case when coupling parameters satisfy the condition $\gamma_{13}=\gamma_{13}^{*}=2 \gamma \Delta_{0} / \Delta_{20}$. In this case the mass of phase density mixed mode is zero $\mu_{0}\left(\gamma_{13}^{*}\right)=0$ and the asymptotic of coupled phase density fluctuation far from the vortex core has power law behavior. We search the deviations of order parameter density and phase from bulk values in the form of power law expansion

$$
\left(\tilde{\Delta}, \tilde{\Delta}_{2}, \tilde{\theta}\right)=\left(C_{\Delta} / r^{p}, C_{\Delta 2} / r^{p_{2}}, C_{\theta} / r^{q}\right)
$$

Substituting this ansatz into the system (910[11) we require that the lowest order terms have the same dependence on $1 / r$. This condition determines the exponents $p=p_{2}=2$ and $q=1$ in (12). Furthermore we obtain the linear system to determine coefficients in Eq.(12)

$$
\begin{gathered}
\frac{\gamma \Delta_{20}}{\Delta_{0}} C_{\Delta}-\gamma C_{\Delta 2}-\gamma_{13} \Delta_{0} C_{\theta}^{2}=g \Delta_{0} \\
\left(\alpha+3 \beta \Delta_{0}^{2}+\gamma_{13}\right) C_{\Delta}-\gamma C_{\Delta 2}-\gamma_{13} \Delta_{0} C_{\theta}^{2}=0 \\
2 \gamma C_{\Delta}-\left(\alpha_{2}+3 \beta_{2} \Delta_{20}^{2}\right) C_{\Delta 2}-\gamma \Delta_{0} C_{\theta}^{2}=0
\end{gathered}
$$

For the parameters employed for numerical calculations we obtain $C_{\Delta}=0.17 \Delta_{0}, C_{\Delta 2}=-0.18 \Delta_{20}$ and $C_{\theta}=$ 1.57 .

\section{Vortex structure near the critical point $\gamma_{13}=\gamma_{13}^{c}$}

The critical point separates regimes in $U(1)$ region with single and double solutions for the vortex structure. The solution with spatial variation of relative phase continuously emerges at $\gamma_{13}>\gamma_{13}^{c}$ where $\gamma_{13}^{c}$ is given by the eigenvalue of linear equation which can be written in the form

$$
\hat{L} \tilde{\theta}=2 \gamma_{13}^{c} \Delta^{2} \tilde{\theta}
$$

where $\tilde{\theta}=\pi-\theta$ and

$$
\hat{L}=-g\left[\Delta^{2} \nabla_{r}^{2}+\left(\Delta^{2}\right)_{r}^{\prime} \partial_{r}\right]+\gamma \Delta_{2} \Delta
$$

is a hermitian operator and therefore has orthogonal eigenfunctions. This form allows to find approximate solution of nonlinear Eq.(9) for small values of $\gamma_{13}^{c}-\gamma_{13}$.

We search the solution of nonlinear Eq.(9) in the form $\tilde{\theta}=A \theta_{\text {lin }}+\Theta$ where $\theta_{\text {lin }}=\theta_{\text {lin }}(r)$ is the normalized eigenfunction of Eq.(16) and $\Theta=\Theta(r)$ is a small correction. It collects the contribution of higher levels of the operator (17) and therefore is orthogonal to $\theta_{\text {lin }}(r)$ so that

$$
\int_{0}^{\infty} r \Delta^{2} \theta_{l i n} \Theta d r=0
$$

To determine the amplitude $A$ we rewrite Eq. (9) in the form

$$
\hat{L} \Theta=2\left(\gamma_{13}^{c}-\gamma_{13}\right) \Delta^{2} A \theta_{l i n}+A^{3} N\left(\theta_{l i n}\right)
$$

where the last term is nonlinear part

$$
N\left(\theta_{\text {lin }}\right)=\left(8 \gamma_{13} \Delta^{2}-\gamma \Delta \Delta_{2}\right) \theta_{\text {lin }}^{3} / 6 .
$$

obtained with the help of Taylor expansion $\sin \theta \approx-\tilde{\theta}+$ $\tilde{\theta}^{3} / 6$. Taking the inner product of both parts of Eq. (19) with $\tilde{\theta}_{l i n}(r)$ and employing the hermiticity of operator $\hat{L}$ and orthogonality (18) we get the amplitude

$$
A=\sqrt{\frac{2\left(\gamma_{13}-\gamma_{13}^{c}\right)}{\int_{0}^{\infty} r \theta_{l i n}^{2} N\left(\theta_{l i n}\right) d r}}
$$


Thus we obtain that at $\gamma_{13}>\gamma_{13}^{c}$ the vortex structure can have two solutions. One is that with constant interband phase $\theta(r)=$ const and the second one is with the phase variation given by the eigenfunction of operator (17) with the amplitude $A \sim \sqrt{\gamma_{13}-\gamma_{13}^{c}}$ given by Eq.(20).

\section{Time-dependent Ginzburg-Landau theory and forces acting on moving vortex in three-component superconductor}

We describe the non-equilibrium process of vortex motion near the critical temperature with time-dependent GL model generalized to a two-gap superconductor

$$
\Gamma_{k}\left(\partial_{t}-i \varphi\right) \psi_{k}=-\delta F / \delta \psi_{k}^{*}
$$

where $j=1,2,3, \varphi$ is the electric potential, $\Gamma_{k}$ are damping constants. The expression for the supercurrent is then $\mathbf{j}_{\mathbf{s}}=2 g_{k} \mathbf{Q}_{k} \Delta_{k}^{2}$ where $\mathbf{Q}_{k}=\nabla \theta_{k}+\mathbf{A}$ and for the normal current $\mathbf{j}_{\mathbf{n}}=\sigma_{n} \mathbf{E}$ where $\mathbf{E}=\nabla \varphi+\dot{\mathbf{A}}$. Note that normal and superconducting current can convert into each other thus they are not separately conserved. For the superfluid current we have an expression $\mathbf{j}_{\mathbf{s}}=-\delta F / \delta \mathbf{A}$ so that $\operatorname{div}_{\mathbf{s}}=i\left(\psi_{k}^{*} \delta F / \delta \psi_{k}^{*}-\psi_{k} \delta F / \delta \psi_{k}\right)$. Hence from Eq.(3) we obtain that divj $\mathbf{j}_{\mathbf{s}}=-2 \Gamma_{k} \Delta_{k}^{2} \Phi_{k}$ where $\Phi_{k}=\left(\varphi-\dot{\theta}_{k}\right)$. Taking into account the total current conservation $\operatorname{div}\left(\mathbf{j}_{\mathbf{s}}+\mathbf{j}_{\mathbf{n}}\right)=0$ we can get the Poisson equation for quasistationary electric field with the electric charge density given by $\rho=-\operatorname{divj}_{\mathbf{s}} /\left(4 \pi \sigma_{n}\right)$ so that

$$
\sigma_{n} \operatorname{div} \mathbf{E}=2 \Gamma_{k} \Delta_{k}^{2} \Phi_{k}
$$

Assuming the Coloumb gauge for the vector potential $\operatorname{div} \mathbf{A}=0$ we obtain the Poisson equation

$$
\sigma_{n} \Delta \varphi=2 \Gamma_{k} \Delta_{k}^{2}\left(\varphi-\dot{\theta}_{k}\right)
$$

which we employ to calculate the distribution of the scalar potential $\varphi$ generated by the moving vortex.

Steady state vortex motion with constant velocity $\mathbf{U}$ is determined by the force balance $\mathbf{f}_{\text {env }}+\mathbf{f}_{\mathbf{L}}=0$ between Lorentz force $\mathbf{f}_{\mathbf{L}}$ acting on the vortex from external transport current and force from the environment $\mathbf{f}_{\mathbf{L}}$.

The force acting on the moving vortices is determined by the variation of free energy due to the small vortex displacement 14, 15] $\delta F=-(\mathbf{f} \cdot \mathbf{d})$. In general the variation of the free energy is

$$
\delta F=\int\left[\frac{\delta F}{\delta \psi_{k}} \delta \psi_{k}+\text { c.c. }+\frac{\delta F}{\delta \mathbf{A}} \delta \mathbf{A}+\frac{\mathbf{B}}{4 \pi} \operatorname{rot} \delta \mathbf{A}\right] d V
$$

The last two terms here can be found using the identity

$$
\frac{\delta F}{\delta \mathbf{A}} \delta \mathbf{A}+\frac{\mathbf{B}}{4 \pi} \operatorname{rot} \delta \mathbf{A}+\operatorname{div}[\mathbf{B} \times \delta \mathbf{A}]=\mathbf{j}_{\mathbf{n}} \delta \mathbf{A}
$$

therefore neglecting the surface term

$$
\delta F=\int\left[\frac{\delta F}{\delta \psi_{k}} \delta \psi_{k}+\text { c.c. }+\mathbf{j}_{\mathbf{n}} \delta \mathbf{A}\right] d V .
$$

Besides the variation of the free energy we take into account the interaction of vortices with transport current $\mathbf{j}_{\text {tr }}$ created by the external source. It is given by

$$
\delta F_{\text {ext }}=-\int \mathbf{j}_{\mathbf{t r}} \delta \mathbf{A} d V
$$

According to the conventional procedure we consider the variation of the free energy due to the vortex displacement described by

$$
\begin{aligned}
\delta \psi_{k} & =(\mathbf{d} \cdot \nabla) \psi_{k} \\
\delta \mathbf{A} & =(\mathbf{d} \cdot \nabla) \mathbf{A} .
\end{aligned}
$$

Lorentz force Now we consider the action of the homogeneous transport current $\mathbf{j}_{\text {tr }}$ on vortex. To calculate the force acting on vortex we evaluate the energy change due the infinitesimal translations of vortex center. Then the elementary work of the external force has the form

$$
\delta F_{\text {ext }}=-\int \mathbf{j}_{\mathbf{t r}} \delta \mathbf{A} d V
$$

where $\delta \mathbf{A}=(\mathbf{d} \cdot \nabla) \mathbf{A}$. Now we use the following identities

$$
\operatorname{div}\left[\mathbf{j}_{\operatorname{tr}}(\mathbf{d} \cdot \mathbf{A})\right]=\mathbf{j}_{\operatorname{tr}}[\mathbf{d} \times \mathbf{B}]+\mathbf{j}_{\mathbf{t r}}(\mathbf{d} \nabla) \mathbf{A}
$$

to obtain

$$
\delta F_{\text {ext }}=\mathbf{d} \int\left[\mathbf{j}_{\mathrm{tr}} \times \mathbf{B}\right] d V=2 \pi \mathbf{d} \cdot\left(\mathbf{j}_{\mathrm{tr}} \times \mathbf{z}_{\mathbf{v}}\right)
$$

where $\mathbf{z}_{\mathbf{v}}$ is the vorticity direction. Therefore the force is

$$
\mathbf{f}_{\mathbf{L}}=2 \pi\left[\mathbf{j}_{\operatorname{tr}} \times \mathbf{z}_{\mathbf{v}}\right]
$$

Force from the environment To calculate the force from the environment we should consider the energy variations due to displacement $\delta \psi_{k}=-\mathbf{d} \cdot \nabla_{\mathbf{r}} \psi_{k}$ and $\delta \mathbf{A}=-\mathbf{d} \cdot \nabla_{\mathbf{r}} \mathbf{A}$. Then we can make use of Eq.(21) which results

$$
\delta F=\int\left[\frac{\delta F}{\delta \psi_{k}}\left(\mathbf{d} \nabla \psi_{k}\right)+c . c .+\mathbf{j}_{\mathbf{n}}(\mathbf{d} \nabla) \mathbf{A}\right] d V
$$

Further we use the fact $\operatorname{div} \mathbf{j}_{\mathbf{s}}=i\left(\psi_{k}^{*} \delta F / \delta \psi_{k}^{*}-\psi_{k} \delta F / \delta \psi_{k}\right)$ and transform the above equation as follows

$$
\begin{aligned}
\delta F=\int\left[\frac{\delta F}{\delta \psi_{k}}(\mathbf{d} \cdot(\nabla-i \mathbf{A})) \psi_{k}+c . c .\right] d V- \\
\\
\int\left[(\mathbf{d} \cdot \mathbf{A}) \operatorname{div} \mathbf{j}_{\mathbf{s}}+\mathbf{j}_{\mathbf{n}}[(\mathbf{d} \cdot \nabla) \mathbf{A}]\right] d V
\end{aligned}
$$

The last two terms can be written using the identity

$$
\mathbf{j}_{\mathbf{n}}[(\mathbf{d} \cdot \nabla) \mathbf{A}]+(\mathbf{d} \cdot \mathbf{A}) \operatorname{div}_{\mathbf{n}}=\mathbf{d} \cdot\left[\mathbf{j}_{\mathbf{n}} \times \mathbf{B}\right]
$$


Therefore neglecting the surface term we get

$$
\delta F=\mathbf{d} \cdot \int\left[\frac{\delta F}{\delta \psi_{k}}(\nabla-i \mathbf{A}) \psi_{k}+\text { c.c. }+\left[\mathbf{j}_{\mathbf{n}} \times \mathbf{B}\right]\right] d V
$$

Now let us make use of the Eqs. (21) to substitute

$$
\begin{aligned}
& \int\left[\Gamma_{k}\left(\partial_{t}+i \varphi\right) \psi_{k}^{*}(\nabla-i \mathbf{A}) \psi_{k}+c . c .\right] d V \\
& =2 \int\left[\Gamma_{k}\left(\nabla \Delta_{k} \dot{\Delta}_{k}+\Phi_{k} \mathbf{Q}_{\mathbf{k}} \Delta_{k}^{2}\right)\right] d V
\end{aligned}
$$

which finally yields

$$
\begin{gathered}
\delta F= \\
\mathbf{d} \cdot \int\left[-2 \Gamma_{k}\left(\nabla \Delta_{k} \dot{\Delta}_{k}+\Phi_{k} \mathbf{Q}_{\mathbf{k}} \Delta_{k}^{2}\right)+\left[\mathbf{j}_{\mathbf{n}} \times \mathbf{B}\right]\right] d V
\end{gathered}
$$

For the typical type-II superconductors the last term is usually neglected. Then we obtain the force acting on the unit length of moving vortex line from the environment

$$
\mathbf{f}_{\text {env }}=2 \int \Gamma_{k}\left(\nabla \Delta_{k} \dot{\Delta}_{k}+\Phi_{k} \mathbf{Q}_{\mathbf{k}} \Delta_{k}^{2}\right) d^{2} r
$$

[1] Y. Kamihara, T. Watanabe, M. Hirano, and H. Hosono, J. Am. Chem. Soc. 130, 3296 (2008).

[2] T. K. Ng and N. Nagaosa, Europhys. Lett. 87, 17003 (2009).

[3] V. Stanev and Z. Tesanovic, Phys. Rev. B 81, 134522 (2010).

[4] J. Carlstrom, J. Garaud, and E. Babaev, Phys. Rev. B 84, 134518 (2011).

[5] Saurabh Maiti, Andrey V. Chubukov, Phys. Rev. B 87, 144511 (2013).
[6] W.-C. Lee, S.-C. Zhang, and C. Wu, Physical Review Letters 102, 217002 (2009); C. Platt, R. Thomale, C. Honerkamp, S.-C. Zhang, and W. Hanke, Phys. Rev. B 85, 180502 (2012).

[7] J.Garaud, J.Carlstrom, and E. Babaev, Phys. Rev. Lett. 107, 197001 (2011); J. Garaud, J. Carlstrom, E. Babaev, M. Speight Phys. Rev. B 87, 014507 (2013).

[8] S.-Z. Lin and X. Hu, Physical Review Letters 108, 177005 (2012).

[9] Troels Bojesen, Egor Babaev, Asle Sudbo, arXiv:1306.2313

[10] M. Silaev, E. Babaev Phys. Rev. B 85, 134514 (2012).

[11] In the phase-only London model this divergence was identified earlier as the massless Leggett mode in [8].

[12] The existence of a diverging length scale at the $Z_{2}$ phase transition and mixing of phase difference and density modes dictates that there should be diverging coherence length at the TRSB phase transition while magnetic field penetration length should stay finite. This in turns implies that if the system should generically have "type-1.5" regime [4] in the vicinity of TRSB state with one coherence length larger and other smaller than the magnetic field penetration length (if the system is not a type-I superconductor). Here we consider the case of vortex lattice (e.g. large fields). Results should also apply to interiour of macroscopically large vortex clusters.

[13] The stability analysis, requires relaxing axially symmetric ansatz. It is beyond the scope of this paper and will be presented elsewhere.

[14] N. B. Kopnin, Theory of nonequlibrium superconductivity, Oxford University Press, (2001).

[15] A. T. Dorsey, Phys. Rev. B 46, 8376 (1992).

[16] J. Bardeen and M.J.Stephen Phys. Rev. 140, 1197 (1965).

[17] M. Tinkham, Introduction to superconductivity, Dover Publications (2004).

[18] D. Weston, E. Babaev arXiv:1306.3179. 